\title{
CMB Power Spectrum Estimation via Hierarchical Decomposition
}

\author{
Olivier Doré ${ }^{1}$, Lloyd Knox ${ }^{2}$, and Alan Peel ${ }^{2}$ \\ 1 Institut d'Astrophysique de Paris, 98bis Boulevard Arago, F-75014 Paris, FRANCE; dore@iap.fr \\ 2 Department of Physics, One Shields Avenue, University of California, Davis, California 95616, USA; \\ lknox@ucdavis.edu,apeel@bubba.ucdavis.edu
}

(October 25, 2018)

\begin{abstract}
We have developed a fast, accurate and generally applicable method for inferring the power spectrum and its uncertainties from maps of the cosmic microwave background (CMB) in the presence of inhomogeneous and correlated noise. For maps with $10^{4}$ to $10^{5}$ pixels, we apply an exact power spectrum estimation algorithm to submaps of the data at various resolutions, and then combine the results in an optimal manner. To analyze larger maps efficiently one must resort to suboptimal combinations in which cross-map power spectrum error correlations are only calculated approximately. We expect such approximations to work well in general, and in particular for the megapixel maps to come from the next generation of satellite missions.
\end{abstract}

98.70.Vc

\section{INTRODUCTION}

The anisotropy of the Cosmic Microwave Background $(\mathrm{CMB})$ is proving to be a powerful cosmological probe [i]. Many cosmological parameters, and the primordial power spectra of density and gravity-wave perturbations, can be inferred from the statistical properties of the CMBin particular from its angular power spectrum [2]. Unfortunately, exact methods for calculating the power spectrum and its uncertainties from real observations are very expensive computationally [3. Supercomputers are required for analysis of current datasets and even they will not be sufficient for the next generation of experiments [4]. Here we introduce an approximate method for reducing a CMB map to a power spectrum and its uncertainties.

Generally applicable exact methods for finding the angular power spectrum, $C_{l}$, that maximize the likelihood have operation counts proportional to $N^{3}$ where $N$ is the number of pixels in the map. Our approach to overcoming this $N^{3}$ scaling involves a hierarchical decomposition of the map into a set of submaps. That is, we subdivide the original ("primary") map into non-overlapping regions, each with a manageable number of pixels, in order to estimate the power spectrum from each of these submaps using an exact algorithm. To study the larger angular scale fluctuations we coarsen the primary map and if the number of these coarse pixels is still too large, we again divide into submaps. To go to yet larger angular scales, we coarsen the map further, etc... Then we calculate the expected correlations between the power spectrum estimates from all different submaps at all different resolution levels in order to optimally average them together. A similar multi-grid technique was recently developed for the reduction of time-ordered CMB data to maps [5].

Several other approaches to overcoming the $N^{3}$ scaling have been tried. These include the "pseudo- $C_{l}$ " method of [6], and the "correlation-function" approach of [7]. We expect these methods to work well in the case of homogeneous noise, but to be significantly sub-optimal for the levels of inhomogeneity expected in planned observational programs. None of these methods has been shown to deal properly with correlated noise. Minor modifications of the correlation-function approach may make this path very attractive, though a remaining issue is the importance of noise correlations between pixels.

The $N^{3}$ scaling has been overcome also by a specialpurpose exact method that is expected to be applicable to the maps generated by NASA's Microwave Anisotropy Probe $(M A P)$ satellite . This method [8] assumes the noise is not correlated from one pixel to another and that the noise level variations are roughly azimuthally symmetric. Some of the techniques used in [8] may eventually find their place in more generally applicable (and yet still exact) power spectrum estimation algorithms, though the feasibility is not yet clear. Another specialpurpose exact algorithm is that of [9], which is applicable to experiments that scan on rings. The main idea is to analyze ring sets instead of maps since both the noise and signal covariance structures are simple on the rings, whereas the noise structure can be complicated in the map space. Although some of its critical hypotheses have not been tested yet on realistic data, the ring-set approach might still be of practical importance since it may provide a useful zeroth-order solution for experiments that nearly scan on rings.

In section II we describe our method in detail. In section III we present the results of an application to a map with ten thousand pixels - comparable to the size of maps coming from long-duration balloon (LDB) flights. In section IV we show results from a map four times larger and discuss prospects for application of our method to even larger maps such as those expected from

${ }^{*}$ http://map.gsfc.nasa.gov/ 
MAP and Planck周. In section V we compare with other methods. In section VI we conclude.

\section{METHOD}

Here we first describe our method in the simplest conceptual terms, and then go on to discuss subtleties which complicate our implementation.

\section{A. From the Likelihood Function to the Quadratic Estimator}

We describe here the use of a quadratic estimator to find the maximum of the likelihood function, and the shape of the likelihood function near that maximum, as described in 10$]$. Time-ordered data from observation of the CMB are usually reduced to a set of pixelized maps $\Delta_{i}, i=1, \ldots N$ which can be decomposed into the sum of a signal and a noise contribution, $\Delta=s+n$. Assuming that both the noise and the signal are normallydistributed, their statistical properties are fully characterized by the covariance matrices $S=\left\langle s s^{T}\right\rangle$ and $N=\left\langle n n^{T}\right\rangle$. Assuming furthermore that the noise and signal are not correlated with each other, we can define

$$
C \equiv\left\langle\Delta \Delta^{T}\right\rangle=S+N .
$$

The observed sky signal is assumed to be the realization of an isotropic Gaussian random field whose power spectrum $C_{l}$ is the quantity we want to measure. Thus we are interested in the likelihood function $\mathcal{L}\left(\Delta \mid C_{l}\right)$ which is given by

$$
-2 \ln \mathcal{L}\left(\Delta \mid C_{l}\right)=\ln \operatorname{det} \mathrm{C}+\Delta^{\mathrm{T}} \mathrm{C}^{-1} \Delta .
$$

In particular we are interested in the location of the maximum of this function (which is the most likely $C_{l}$ ) and the curvature at the maximum, $-\partial^{2} \ln \mathcal{L} / \partial C_{l} \partial C_{l^{\prime}}$ (which is approximately the inverse of the covariance matrix for $C_{l}$ ). Note that $C$ depends on $C_{l}$ since

$$
S_{i i^{\prime}}=\sum_{l} \frac{2 l+1}{4 \pi} C_{l} \mathcal{W}_{i i^{\prime}}(l)
$$

where $\mathcal{W}$ is the covariance window function of the experiment.

Given an initial estimate of $C_{l}$ (hereafter, the input $C_{l}$ ) one can reach the likelihood maximum as follows. By Taylor-expanding $\ln \mathcal{L}$ to second order in $\delta C_{l}$ around $C_{l}$, and replacing $-\partial^{2} \ln \mathcal{L} / \partial C_{l} \partial C_{l^{\prime}}$ with its expectation value one can find an expression for $\delta C_{l}$ such that $C_{l}+\delta C_{l}$ maximizes the likelihood:

\footnotetext{
${ }^{\dagger}$ http://astro.estec.esa.nl/SA-general/Projects/Planck/
}

$$
\delta C_{l}=\sum_{l^{\prime}} \frac{1}{2} F_{l l^{\prime}}^{-1} \operatorname{Tr}\left[\left(\Delta \Delta^{\mathrm{T}}-C\right)\left(C^{-1} \frac{\partial C}{\partial C_{l^{\prime}}} C^{-1}\right)\right]
$$

and

$$
F_{l l^{\prime}}=\frac{1}{2} \operatorname{Tr}\left[C^{-1} \frac{\partial C}{\partial C_{l}} C^{-1} \frac{\partial C}{\partial C_{l^{\prime}}}\right]
$$

is the Fisher matrix [11].

Equation 1 is a quadratic function of the data and hence the expression "quadratic estimator". Note that we have suppressed the pixel indices in the various vectors and matrices. Since $\ln \mathcal{L}$ is not equal to its secondorder Taylor expansion (i.e., $\mathcal{L}$ is not a Gaussian in $C_{l}$ ), some iteration is generally required to reach the likelihood maximum.

\section{B. Hierarchical Decomposition and Recombination}

Now let us consider multiple maps and use Greek indices to label them. Estimates of $\delta C_{l}$ from map $\alpha, \delta C_{l}^{\alpha}$, are correlated with those from map $\beta$ with correlation matrix:

$$
\begin{aligned}
\left\langle\delta C_{l}^{\alpha} \delta C_{l^{\prime}}^{\beta}\right\rangle \equiv & \mathcal{F}_{\alpha l, \beta l^{\prime}}^{-1} \\
= & \sum_{l^{\prime \prime}, l^{\prime \prime \prime}}\left(F_{\alpha}^{-1}\right)_{l l^{\prime \prime}}\left(F_{\beta}^{-1}\right)_{l^{\prime} l^{\prime \prime \prime}} \times \\
& \frac{1}{2} \operatorname{Tr}\left[A_{\alpha, l^{\prime \prime}} C_{\alpha \beta} A_{\beta, l^{\prime \prime \prime}} C_{\beta \alpha}\right]
\end{aligned}
$$

where

$$
A_{\alpha, l} \equiv C_{\alpha \alpha}^{-1} \frac{\partial C_{\alpha \alpha}}{\partial C_{l}} C_{\alpha \alpha}^{-1} .
$$

Note that if $\alpha=\beta$ then Eq. 6 simplifies to the usual result:

$$
\left\langle\delta C_{l} \delta C_{l^{\prime}}\right\rangle=F_{l l^{\prime}}^{-1} .
$$

Given this result, we know how to combine the various $\delta C_{l}$ estimates from each submap into a final $\delta C_{l}$ estimate from all the submaps in a minimum-variance (optimal) manner. The minimum-variance $\delta C_{l^{\prime}}$ satisfies

$$
\sum_{l^{\prime}}\left(\sum_{\alpha \beta} \mathcal{F}_{\alpha l, \beta l^{\prime}}\right) \delta C_{l^{\prime}}=\sum_{\alpha \beta l^{\prime}} \mathcal{F}_{\alpha l, \beta l^{\prime}} \delta C_{l^{\prime}}^{\alpha}
$$

and has a weight matrix (inverse of covariance matrix):

$$
F_{l l^{\prime}}=\sum_{\alpha \beta} \mathcal{F}_{\alpha l, \beta l^{\prime}}
$$

Although for simplicity we have written these expressions for estimating individual $C_{l}$ 's, issues of signal-tonoise and spectral resolution usually lead us to estimate the power spectrum in bands of $\ell$, where the shape of 
$C_{l}$ inside the bands is assumed. The usual assumption (which we use in our applications) is that $l(l+1) C_{l} /(2 \pi)$ is constant inside the band.

Our treatment of the correlations of the $\delta C_{l}$ 's between pairs of maps has been general. The maps may be spatially separate or overlapping; they may have equivalent or different pixel sizes. Thus we have worked out the most general solution to optimally combine the power spectra of submaps which are the result of hierarchical decomposition (HD) of a primary map.

\section{Spectral Resolution}

Even with optimal combining of the power spectrum estimates from the various submaps, the HD procedure results in a sub-optimal estimation of the power spectrum. Fortunately, in the cases we study, the suboptimal results are quite close to the optimal results. Departure from the optimal results is almost entirely due to the degraded spectral resolution of the high-resolution submaps. This loss of spectral resolution is the primary drawback of the HD approach.

The spectral resolution is most severely degraded at the highest resolution levels where the submaps have the smallest spatial extents. For any map of linear extent, $L$, it is difficult to distinguish the mode $P_{l}(\cos \theta)$ from $P_{l+\delta l}(\cos \theta)$ where $\delta l \lesssim \pi / L[12$. If one wishes to achieve a spectral resolution of $\delta l$ for a square map with linear pixel size $r_{p}$ then this map must have $n$ pixels where

$$
n \simeq 2.5 \times 10^{3}\left(\frac{30}{\delta l} \frac{7^{\prime}}{r_{p}}\right)^{2} .
$$

Fortunately $\delta l=30$ and $r_{p}=7^{\prime}$ are expected to be adequate for LDB-type maps and $2.6 \times 10^{3}$ pixels is a small enough submap size to allow for reasonable computation times (as shown below).

\section{Scaling}

We now calculate how computation time scales with total number of pixels in the full-resolution primary map, $N$, and the number of multipole-moment bands, $N_{b}$. For simplicity we assume that all submaps have the same number of pixels, $n$, and that we estimate the power spectrum for each submap in the same number of bands. Estimating the power spectrum and Fisher matrix for each submap takes on the order of $N_{b}^{2} n^{3}$ operations so at the finest level we have on the order of $N_{b}^{2}(N / n) n^{3}=N_{b}^{2} N n^{2}$ operations. In a systematic coarsening (such as the one below defined by combining four pixels at one resolution into one larger pixel for the next coarser level), most of the submaps are at the finest resolution and therefore analysis and combining of these finest submaps dominates the demands on memory and CPU time.
For large enough $N$, the dominant computational step will be in calculating the correlations between submaps. The matrix multiplication in Eq. 6 takes on the order of $n^{3}$ operations, so performing it for every pair of submaps and pair of bands takes on the order of $N_{b}^{2} N^{2} n$ operations.

The procedure can in principle be parallelized for the efficient use of $n_{\text {proc }}$ processors, where $n_{\text {proc }}$ ranges anywhere from $N_{b}$ to $\sim(N / n)^{2} N_{b}^{2}$. The crucial use of parallelization comes in the dominant combining stage, which scales as $(N / n)^{2} N_{b}^{2}$, and involves the combination of $\sim \frac{1}{2}(N / n)^{2}$ pairs of submaps. This type of independent pair loading can be efficiently shared on any number of processors lower than $\frac{1}{2}(N / n)^{2}$. For LDB-type missions one might have $N_{b} \sim 10$ and $(N / n)^{2} N_{b}^{2} \sim 4 \times 10^{4}$ and approximately 200 pairs of submaps to combine (if we indiscriminately retain all submap-submap correlations (see Section IV)). For supercomputers with $n_{\text {proc }} \lesssim 10^{2}$, every processor can be efficiently used.

\section{E. The Noise Matrix}

Our approach assumes that we begin with a pixelized map and its corresponding noise covariance matrix. Map-making procedures usually produce a weight matrix, which is the inverse of the noise matrix. Inverting an arbitrary weight matrix takes on the order of $N^{3}$ operations. Fortunately, this inversion only needs to be done once and is feasible for LDB-size maps.

For larger maps, treatment of the weight matrix by general matrix inversion algorithms is impossible. Fast methods are being developed [13] which rely on the origin of the map weight matrix in the weight matrix of the time-ordered data. That is, the map weight matrix is $A^{T} N^{-1} A$ where $N_{t t^{\prime}}^{-1}$ is (here) the time-stream weight matrix for a stationary noise process, and $A_{t i}$ is the pointing matrix element that is one if at time-sample $t$ the telescope is sampling map pixel $i$ and zero otherwise. This special structure allows for each iteration of a conjugate gradient solution to be performed much faster than for an arbitrary matrix.

Another possibility (suggested in [7]) is to calculate the noise covariance matrix by Monte Carlo methods. In other words, one would make repeated simulations of the map noise and average those together to get any desired elements of the noise matrix. In addition to possible speed advantages, this approach also has storage advantages since one probably needs fewer than $N / 2$ realizations to have a sufficiently accurate estimate of the noise. One may still need thousands of realizations of the noise - e.g., 20,000 realizations are required for the noise matrix elements to be accurate to within $1 \%$ of the diagonal. 


\section{F. Coarsening}

The amount of work to be done depends on the choice of number of resolution levels, which is governed by how many pixels are combined to form one pixel at the nextcoarsest level. Greater coarsening between levels leads to fewer required operations, but at the expense of greater loss of information. Since the cost in computing time is slight for using the most modest coarsening possible while maintaining (roughly) square pixels, we always coarsen by averaging four pixels into one. This coarsening is also easily implemented in the HEALPix pixelization scheme, which we use 14.

In general, one can create a coarse submap $\Delta$ from a fine submap $\delta$ as follows:

$$
\Delta=W^{-1} \alpha w \delta
$$

where $\alpha_{c i}$ is one for all fine pixels $i$ in coarse pixel $c$ and zero otherwise, $w$ is some weighting of the fine pixels and $W=\alpha w \alpha^{T}$. The coarse-fine and coarse-coarse noise covariance matrices are given by:

$$
\begin{aligned}
\left\langle\Delta \delta^{T}\right\rangle_{\text {noise }} & =W^{-1} \alpha w N \\
& =W^{-1} \alpha \quad\left(\text { if } w=N^{-1}\right) \\
& =\frac{N \alpha}{4} \quad(\text { if } w=I)
\end{aligned}
$$

and:

$$
\begin{aligned}
\left\langle\Delta \Delta^{T}\right\rangle_{\text {noise }} & =W^{-1} \alpha w N w^{T} \alpha^{T} W^{-1} \\
& =W^{-1} \quad\left(\text { if } w=N^{-1}\right) \\
& =\frac{\alpha N \alpha^{T}}{16} \quad(\text { if } w=I) .
\end{aligned}
$$

For optimal coarsening $w=N^{-1}$ and for uniform averaging, $w=I$. We assume that we are coarsening four pixels into one and therefore that $\alpha \alpha^{T}=4 I$. We see that uniform averaging leads to noise covariance matrices that are easy to calculate. For optimal averaging we need to invert $W$ which is substantially less challenging than inverting $N^{-1}$ to get $N$ since it has $1 / 16$ the number of elements. The same technique used for calculating $N$ by exploiting the origin of $N^{-1}$ in time-ordered data (as explained in the previous subsection) can be used to get $W^{-1}$. 15 .

Coarsening will usually result in pixel sizes that are large compared to the angular resolution of the instrument and therefore pixelization effects must be taken into account. Our treatment of the effect of pixelization on the signal correlation function is approximate, i.e., we use a pixel window which is the average of the evaluated power spectrum for every individual pixel. To prevent these approximations from creating errors in the final power spectrum, we ignore information from multipole moments greater than some critical value where the approximation introduces significant error. Pixelization effects are discussed in more detail in the Application section.

\section{G. Iteration}

A single application of the quadratic estimator of Eq. A might not result in a $C_{l}$ that is sufficiently close to the likelihood maximum. This will be the case if the input $C_{l}$ is too far from the likelihood maximum. Fortunately, iterative application of Eq. 4 has been shown to converge quite rapidly 10 .

When using the hierarchical decomposition approach, it is important that the iteration be done globally. That is, within each iteration, the power spectrum from each submap should be estimated using the same input $C_{l}$. If iteration is performed within the submaps, the combined result will suffer from cosmic bias [16], which results from the fact that uncertainties in $C_{l}$ are not normallydistributed. For a normally-distributed variable, the curvature of the log of the likelihood function is independent of location in the parameter space (because the likelihood is a Gaussian). However, for $C_{l}$, this curvature does depend on location. For larger values of $C_{l}$ the curvature is smaller (i.e., the variance is larger). Thus, upward fluctuations should result in larger variances than downward fluctuations and so if one combines them together assuming Gaussianity, the net result is a downward bias due to the over-weighting of the downward fluctuations.

The combination procedure of Eq. 9 implicitly assumes the estimates are normally distributed. We avoid the cosmic bias that might result from this assumption by weighting the downward and upward fluctuations equally. That is, we make sure to calculate $\mathcal{F}_{\alpha l, \beta l^{\prime}}$ from the same $C_{l}$ for all submaps. Thus any desired iteration, e.g., motivated by a large correction from the input $C_{l}$, should be done globally.

Since the uncertainty in $C_{l}$ is non-Gaussian, specifying the $C_{l}$ that maximizes the likelihood function, and $\left\langle\delta C_{l} \delta C_{l^{\prime}}\right\rangle$, does not completely characterize the uncertainty. The uncertainty can be approximately characterized by use of the "offset $\log$-normal form" [16]. That is, error in the quantity $Z_{l} \equiv \ln \left(C_{l}+x_{l}\right)$ is approximately normally-distributed. The offset, $x_{l}$, is a measure of the noise contribution to the uncertainty, as opposed to the sample-variance contribution to the uncertainty. It can be calculated as outlined in 16 .

\section{APPLICATION}

First we discuss the specifications for the simulated maps we used. Then we compare the results of HD with those of the exact method.

\section{A. Simulation Map Details}

We have applied our method using a Fortran code, which we have named Madcumba, to two different simulated maps, hereafter simulations $A$ and $B$. In both cases, 
the angular-power spectrum used was that of a $C O B E-$ normalized adiabatic, scale-invariant "lambda" cold dark matter $(\Lambda \mathrm{CDM})$ model with $\Omega_{\Lambda}=0.6, \Omega_{b}=0.05$, $\Omega_{\mathrm{cdm}}=0.35$ and $H_{0}=75 \mathrm{~km} \mathrm{sec}^{-1} \mathrm{Mpc}^{-1}$ and was generated by the publicly available code CMBfast 17]. The simulated signal maps were generated using the synfast routine in the publicly available HEALPix package [14], at HEALPix $N_{\text {side }}=256$ (level 8 , where $\left.N_{\text {side }}=2^{\text {level }}\right)$, in which the pixel solid angle is around $\left(13.7^{\prime}\right)^{2}$, assuming a circular beam with full-width at half-maximum of $20^{\prime}$. Finally, pixel noise taken from a Gaussian distribution with zero correlations between pixels was added to the maps. The only significant differences between our two simulations are size and noise characteristics.

The simulation $A$ map has $10^{4}$ pixels, is square in shape, and has a homogeneous noise variance of $(20 \mu K)^{2}$ in each pixel. Its relatively small size allows for the power spectrum to be estimated by the exact method (i.e., without dividing into submaps) using the MADCAP package [4. This is compared to our calculation via HD into four equal-area square 2500 pixel submaps at full resolution and one coarse 2500 pixel submap at HEALPix $N_{\text {side }}=128$ (level 7) which covers the same area as the primary map.

The simulation $B$ map is also square in shape and has $4 \times 10^{4}$ pixels with a noise variance that is cosinemodulated throughout the map, varying from $(20 \mu K)^{2}$ to $9 \times(20 \mu K)^{2}$. Here, we decompose the primary map into sixteen submaps at full resolution, four submaps at the next coarser resolution and one coarsest resolution submap which covers the same area as the primary map but, by being two levels coarser, contains $1 / 16^{\text {th }}$ as many pixels. Thus, as with simulation $A$, we use $n=2500$ pixel submaps.

\section{B. Comparison with Exact Method}

The top panel of Fig. 1 shows estimates of the powers from the individual submaps in simulation $A$. The bottom panel shows both the result of optimally combining them and the exact results obtained directly from the primary map. The solid line in both panels is the original power spectrum for the simulations. The differences between the power estimates are less than $20 \%$ of the standard error from the exact method.

Not only do the power spectrum estimates agree quite well, but so do the estimates of the uncertainties. The error bars in Fig. 1 are the square roots of the diagonal elements of the respective Fisher matrices. In Fig. 2 one can see how well entire rows of the exact and HD Fisher matrices agree.

Clearly, the bigger the submaps at the finest resolution, the better this approach works. For a fixed length scale of interest, larger submaps contain a greater fraction of corresponding pixel pairs, and therefore achieve better spectral resolution $(\delta \ell)$. Unfortunately, the compute- time, when dominated by the combine procedure, scales as $n$ and therefore as $1 / \delta \ell^{2}$ (or possibly $n^{2}$ but with a much smaller pre-factor (see section IV)). Thus, choice of $n$ can be critical. We studied how our information loss varies with $n$ by comparing the error bars from the HD procedure to the full analysis for $n=2500$ (the case above), $n=1600$, and $n=900$. The results are shown in Fig. 3. Note that for the $n=2500$ case all the error bars are increased over the exact case by less than $10 \%$. These larger error bars are consistent with the less than $20 \%$ differences (in units of variance of exact results) between the power estimates.

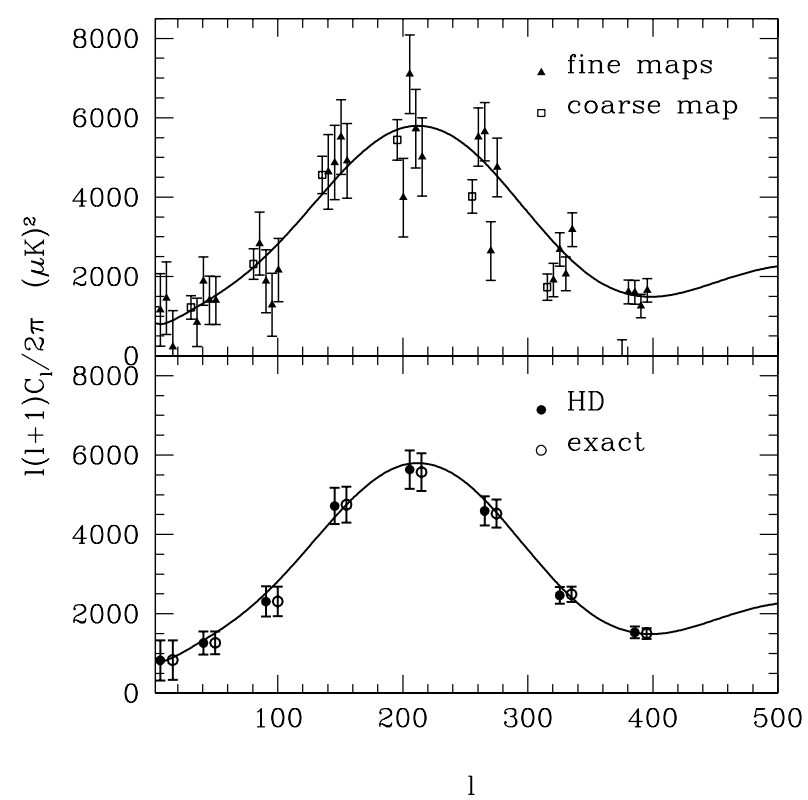

FIG. 1. Simulation $A$ Results. Top panel: Power spectrum estimates from four individual full-resolution 2500 pixel submaps (triangles) and one coarse 2500 pixel submap. Bottom panel: Power spectrum estimates from optimally combining the top-panel results (solid circles) and from the exact calculation (open circles). Note that in both panels, points are slightly shifted horizontally for clarity.

The upward trend in error ratio with increasing band number is an effect of decreasing spectral resolution. To understand this, we examine Fig. 1 which shows the ratio of the HD over the exact method of the band contributions to the total weight, $W_{b}$, where:

$$
W_{b} \equiv \sum_{b^{\prime}} F_{b b^{\prime}}
$$

and the total weight of an experiment is $W \equiv \sum_{b} W_{b}$. For this analysis we switch to a finer binning of 25 bands, each with width $\delta l=30$.

Note first the short-dashed line which is four times the ratio of $W_{b}$ for one full resolution submap over the one for the primary map. If the four submaps were uncorrelated, we would expect this ratio to be $\sim 1$. However, since the 
submaps are correlated, this ratio is greater than 1 . We see that submap-submap correlations are more important at lower $\ell$ than higher $\ell$ values.

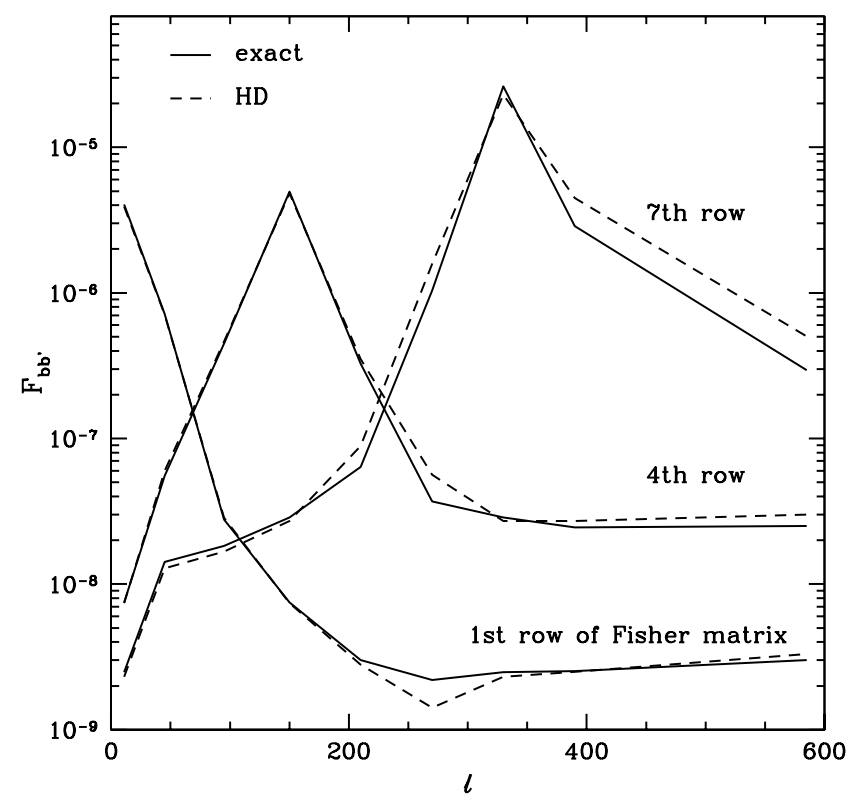

FIG. 2. Three rows of the Fisher matrix calculated exactly (solid lines) and also via the combination (HD) procedure (dashed lines) for simulation $A$.

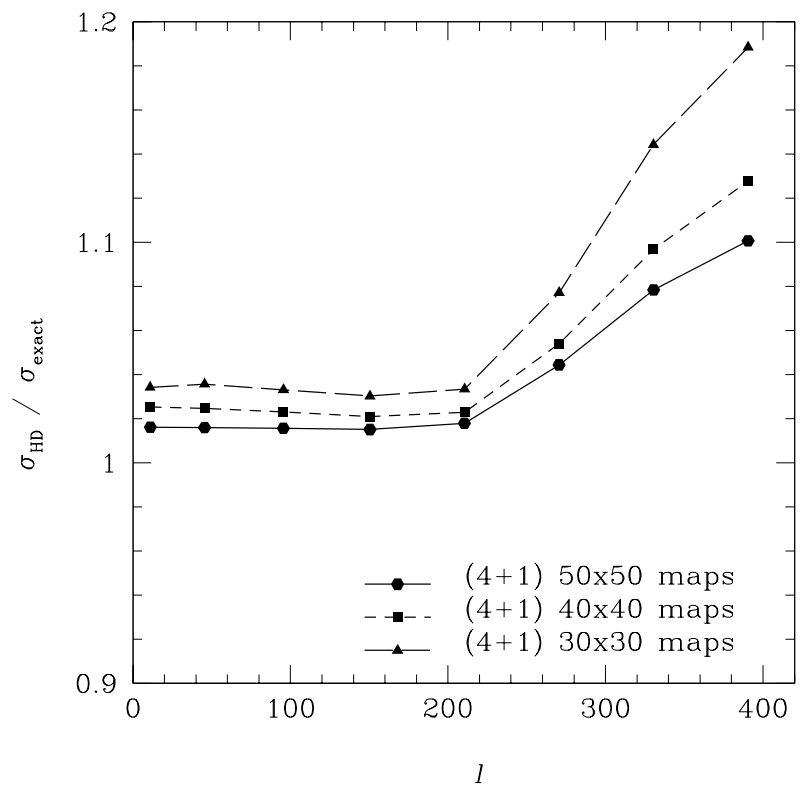

FIG. 3. Error bars from HD divided by error bars for the exact analysis. Each case represents a primary map with $4 n$ pixels divided into four $n$-pixel full resolution submaps and one coarsened $n$-pixel map where $n=30 \times 30$ (triangles), $40 \times 40$ (squares) or $50 \times 50$ (hexagons) .
Though individual elements of the Fisher matrix may be larger for a sub-optimal method than an optimal one, we know that the contribution from a given band to the total weight can not be larger. Thus, the best we could hope for is that the ratio of $W_{b}$ for the HD method over the exact method is near unity. We see from Fig. 4 that it is everywhere greater than 0.97 . Thus the fact that the combine procedure gives at most $10 \%$ larger error bars (20\% larger variances) in Fig. 3 can not be due to any reduction in the total weight (which we see is negligible), but must be due to how each $W_{b}$ is distributed among the $F_{b b^{\prime}}$. In particular, it is the lower spectral resolution of the smaller submaps which results in the $W_{b}$ being more spread out within a Fisher matrix row and less concentrated in the diagonal element $F_{b b}$ as is clear from the 7 th row plotted in Fig. 2.

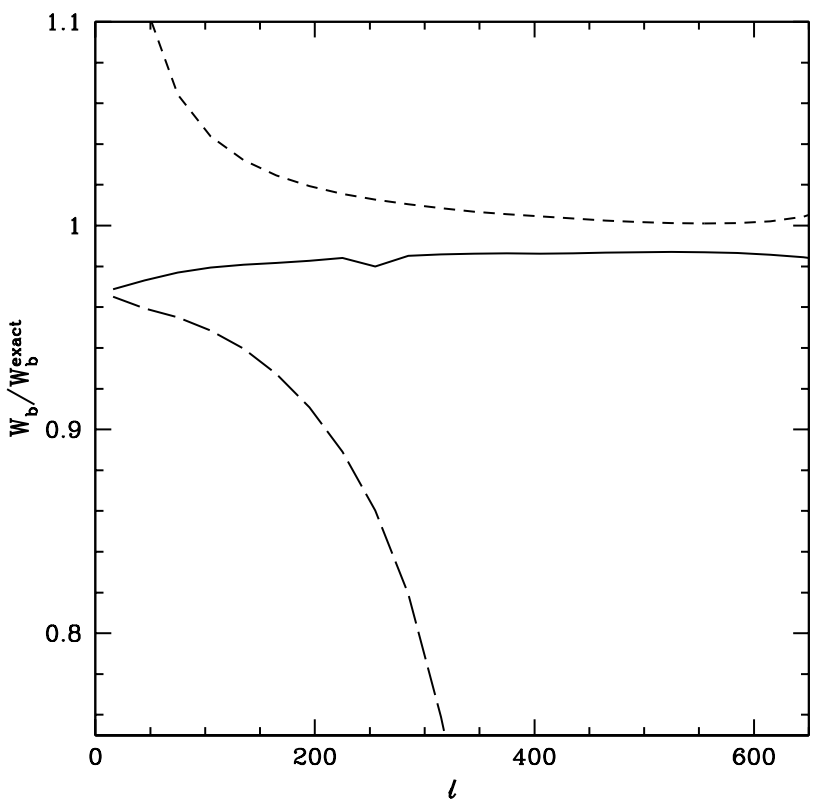

FIG. 4. $W_{b} / W_{b}^{\text {exact }}$ where $W_{b}=\sum_{b^{\prime}} F_{b b^{\prime}}$. The $W_{b}$ 's are from analysis of the simulation $A$ map, but into finer bins of width $\delta l=30$. The short-dashed line is $4 W_{b} / W_{b}^{\text {exact }}$ where $W_{b}$ is just from analysis of one of the four full-resolution submaps; the long-dashed line is $W_{b} / W_{b}^{\text {exact }}$ where $W_{b}$ is from analysis of the coarse resolution submap; the solid line is $W_{b} / W_{b}^{\text {exact }}$ where $W_{b}$ is from combining information from all five submaps.

A plot of $F_{b b}$ ratios (similar to the $W_{b}$ ratio plot of Fig. (4) shows that the cost of this weight redistribution within a Fisher matrix row is a decrease in the diagonal Fisher elements (in the $\ell=250$ to $\ell=600$ range) to $80-85 \%$ of the exact ones. Not only is $F_{b b}$ suppressed then, but the larger off-diagonal elements also lead to larger diagonal elements of $F^{-1}$. With broader bands (such as those used for Fig. 3), the error-bar increase due to degraded spectral resolution is not as severe. The effect of the larger off-diagonal elements propagates from 
band-to-band and is least significant at the lower bands which are benefiting from the full spectral resolution of the coarse submap.

Also in Fig. 4 one can see that the pixelization effects can be fairly severe. This is unfortunate since we only treat the pixelization influence on the signal-correlation matrix, $S$, approximately. Our treatment is that provided with the HEALPix package, which assumes that the correlation between two pixels only depends on the angular distance between them and not on their orientation. This is an approximation for two reasons: the pixels are anisotropic, and their shapes depend on their location. The validity of the approximate window-function can vary from submap to submap if the submaps are not large enough to have a representative sampling of all pixel shapes. This is another reason to use large submaps. We take each cross-level pixel window function to be the geometric mean of the two auto-level pixel window functions.

Because our treatment of pixelization effects is approximate, we throw out information from coarse submaps at a conservatively low $\ell$ value. In simulation $A$, for example, powers from the coarse resolution submap were only considered for $\ell<225$. In the final combined results, the higher bands only use information from the four fine resolution submaps. We eliminate the influence of the coarse submap on the higher bands by inserting very large num-

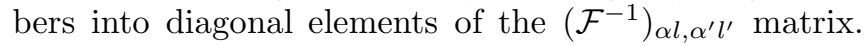
This marginalization technique is described in Appendix A of [10] and can be understood as artificially adding some noise to these particular bands so as to give them very low weight.

The upturn in Figure 3 after $\ell=225$ where the coarse submap information is no longer used indicates that there may be an advantage to keeping the coarse submap information to yet higher $\ell$. This would require a more accurate treatment of the pixel effect on the signal correlation function and its derivatives with respect to $C_{l}$. One way to do this, which would be fairly easy to implement and not cause significant speed reduction, would be to avoid using pixel window functions by calculating coarsened signal matrices directly from finer ones. For example, if the fine signal matrix is $s$ then the next-coarser signal matrix, $S$, must be

$$
S=\frac{\alpha s \alpha^{T}}{16}
$$

where $\alpha_{c i}$ is one for all fine pixels $i$ in coarse pixel $c$ and zero otherwise. Once again, we are summing four pixels into one. The only approximations here come from approximations made in calculating $s$. If these approximations were acceptable for the finer level, they will certainly be adequate for the coarser level. Keeping the coarse level information out to higher bands may be very important for extension to megapixel maps because it is the only other way to improve spectral resolution besides increasing $n$ for the highest-resolution submaps.

\section{ANALYSIS OF GENERAL MEGAPIXEL MAPS}

The map from simulation $A$ has homogeneous white noise. Below we will discuss results from HD analysis of the map from simulation B in which the noise is inhomogeneous but still uncorrelated. Yet we believe HD will work well on realistic maps with correlated noise. In this section we briefly make the case for the success of $\mathrm{HD}$ in the presence of correlated noise and then move on to discuss how HD can be made to work for primary maps with 100 to 1000 times more pixels than the simulation $A$ map. We will see that further approximations are necessary, but that they are likely to work well.

Even though our applications of HD have only been on simulated maps with uncorrelated noise, we believe that HD will work well on realistic maps with correlated noise. This is easiest to see for correlations on length scales smaller than the size of the smallest submaps. Longerrange noise correlations will not be treated accurately in the analysis of the smallest submaps. But this does not matter because the effect will only be on lower- $\ell$ bands where the smallest submaps do not have much weight. The affected bands will be those determined by coarser and larger submaps that will once again be large compared to the correlation length. Thus the prospects for HD on maps with correlated noise are quite good.

Applying HD as we have described it to megapixel maps is prohibitively expensive in terms of the demand on computing resources. A rough scaling argument is sufficient to demonstrate this point. In the megapixel regime, we are strongly dominated by the calculation of all the elements of $\mathcal{F}_{\alpha l, \alpha^{\prime} l^{\prime}}^{-1}$. The number of elements in this matrix is $\sim(N / n)^{2} N_{b}^{2}$. On an SGI Origin 2000, the calculation of a single element of $\mathcal{F}_{\alpha l, \alpha^{\prime} l^{\prime}}^{-1}$ takes $188 \mathrm{sec}$ $(n / 2500)^{3}$ on a single MIPS R12000 $300 \mathrm{MHz}$ processor where $n$ is the number of pixels in a submap. Thus the wall-clock time is

$$
t \sim 1 \text { year }\left(\frac{500}{n_{\text {proc }}}\right)\left(\frac{N}{3 \times 10^{6}}\right)^{2}\left(\frac{n}{2500}\right)\left(\frac{N_{b}^{2}}{1000}\right)
$$

where we have assumed the efficient use of $n_{\text {proc }}$ processors 18. Thus the need to avoid exact calculation of every element of $\mathcal{F}_{\alpha l, \alpha^{\prime} l^{\prime}}^{-1}$ is apparent.

To make the case for the likely success of fast approximations to $\mathcal{F}_{\alpha l, \alpha^{\prime} l^{\prime}}^{-1}$ we turn to the results from simulation $B$. In Fig. 5, we plot four power spectra: one is the result of optimally combining the individual power spectra; one is the power spectrum of the coarsest submap; the other two are the result of a simple averaging of the power spectra for the submaps within a given resolution level as if they were independent. Again, the solid line represents the original input power spectrum.

We find the very good agreement between simple averaging and the exact combination (for the highest bands) to be very encouraging because it is strong evidence that 
signal correlations between non-overlapping submaps are not very important. We certainly see they are not important in the highest bands which are influenced only by submaps with no spatial overlap (since the submaps are all at the same resolution level). If any given band is only influenced by at most two or three levels and we only need to calculate correlations for non-zero submaps then the vast majority of submap pairs can be ignored. Even if some cannot be ignored, their relative insignificance means that there are probably crude approximations to them that will work well.

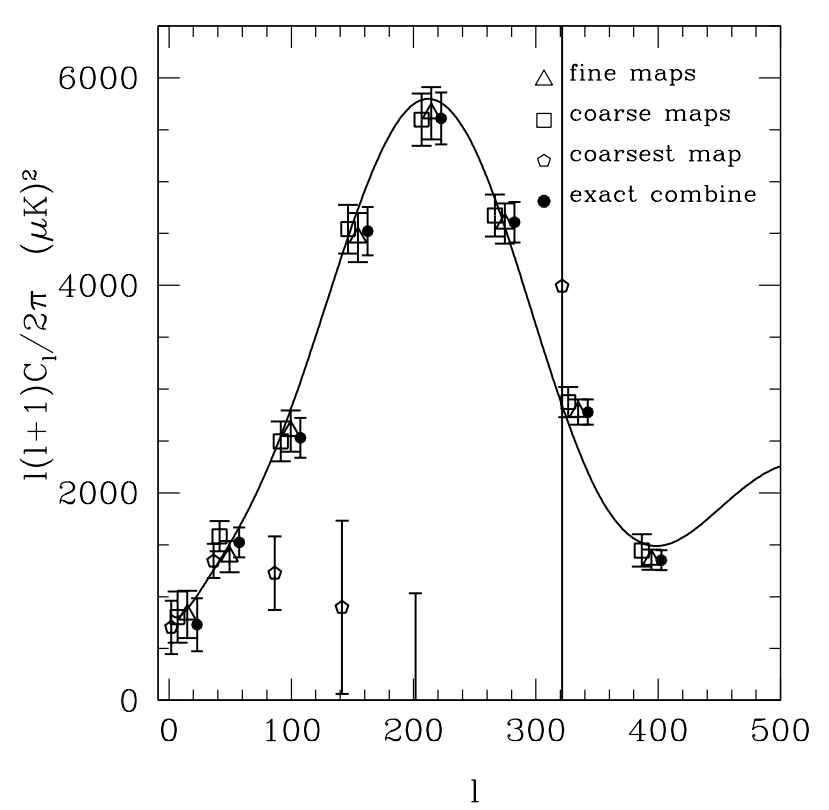

FIG. 5. Results from HD of the 200 by 200 pixel simulation $B$ map. There are sixteen submaps at the finest resolution level (level $8\left(13.7^{\prime}\right)$ pixels), four at the medium level (level $7\left(27.5^{\prime}\right)$ pixels) and one at the coarsest level (level $6\left(55^{\prime}\right.$ pixels)). Triangles and squares represent the result of doing a naively weighted average of the power spectrum estimates, i.e., neglecting correlations, from the sixteen fine submaps and the four coarse submaps, respectively. Pentagons represent results for the one coarsest submap. Filled circles show the results of the optimal combination of all submaps in which all power-spectrum correlations are computed exactly. As in Fig. 1, points are shifted horizontally for clarity.

Calculating only the correlations between overlapping submaps at adjacent resolution levels takes time

$$
t=78 \text { hours }\left(\frac{200}{n_{\text {proc }}}\right)\left(\frac{N}{10^{7}}\right)\left(\frac{n}{5000}\right)^{2}\left(\frac{N_{b}}{40}\right)\left(\frac{\Delta N_{b}}{3}\right)
$$

where for each of the $N_{b}$ bands only the nearest $\Delta N_{b}$ bands are considered [19]. Calculating correlations between overlapping submap pairs whose resolution levels differ by 2 will, at most, double the time. Correlations between non-overlapping map pairs may be significant but can probably be treated approximately in an insignificant amount of time. Development and study of these approximations is probably necessary for practical application of HD to megapixel maps.

We also see from Fig. 5 that even when there is a mix of resolution levels influencing a band, using just one of those levels provides a rough approximation. A fairly good "quick-and-dirty" power-spectrum estimator is the coarsest submap's power spectrum for band 1 , the coarse submaps' power spectrum for bands 2 and 3 , and the finest submaps' power spectrum for bands 4 to 8 . Such an estimator has its applications, for example, finding a $C_{l}$ that is close enough to optimal that one only needs a single iteration of the exact HD procedure.

The scaling of $t$ with $N$ in Eq. 18 is linear if $n$ is fixed. But if we fix spectral resolution and the area of the primary map, then $n \propto N$ and therefore $t \propto N^{3}$ once again! Or, at fixed $N$ and primary map area, $t \propto(1 / \delta l)^{4}$. Our fiducial choice above of $n=5000$ corresponds for Planck with $N=10^{7}$ and $r_{p}=3.5^{\prime}$ to $\delta_{l}=45$. This may be sufficient since physical models have fairly smooth powerspectra. We see that the degree to which degraded spectral resolution affects our ability to discriminate between different models is a crucial issue for the applicability of HD to Planck. We remind the reader that spectral resolution is the only thing that is significantly compromised with HD; Fig. 4 shows the total weight from each band is within a few percent of optimal.

\section{COMPARISON WITH OTHER METHODS}

The HD method has many advantages over other fast, approximate methods. Perhaps the chief advantage is its ability to handle maps with correlated noise. Its main disadvantage is spectral resolution. To understand better these competitive advantages/disadvantages it is worth spending some time discussing these other methodsespecially since we will see they are somewhat complementary and hence a hybrid approach may be useful.

This discussion of other methods is facilitated by writing down the following generalization of Eq. 4 :

$$
C_{l}=\sum_{l^{\prime}} \frac{1}{2} F_{l l^{\prime}}^{-1} \operatorname{Tr}\left[W\left(\Delta \Delta^{\mathrm{T}}-N\right) W \frac{\partial C}{\partial C_{l^{\prime}}}\right]
$$

and Eq. 5 ;

$$
F_{l l^{\prime}}=\frac{1}{2} \operatorname{Tr}\left[W \frac{\partial C}{\partial C_{l}} W \frac{\partial C}{\partial C_{l^{\prime}}}\right] .
$$

These equations specify a general unbiased quadratic estimator, with pixel pair-weighting determined by $W$. The $F_{l l^{\prime}}$ matrix is derived by demanding that the estimator be unbiased $\left(\left\langle C_{l}^{\text {estimate }}\right\rangle=C_{l}\right)$. In general, its inverse is not equal to $\left\langle\delta C_{l} \delta C_{l^{\prime}}\right\rangle$ which is instead given by 


$$
\mathcal{F}_{l l^{\prime}}^{-1}=\frac{1}{2} F_{l l^{\prime \prime}}^{-1} F_{l^{\prime} l^{\prime \prime \prime}}^{-1} \operatorname{Tr}\left[A_{l^{\prime \prime}} C A_{l^{\prime \prime \prime}} C\right]
$$

where $A_{l} \equiv W \frac{\partial C}{\partial C_{l}} W$, similar to Eq. 17.

For the minimum-variance estimator, $W=C^{-1}$. The "correlation-function" approach (CF) of [7] uses the simpler $W=I$ in pixel space [20]. Spherical-harmonic transforming the map and averaging $\left|a_{l m}\right|^{2}$ 's over $m$ uses $W=I$ in spherical-harmonic space. The multiscale method we have just described above likewise corresponds to a choice of $W$, although this $W$ is not easily written down.

It is worth pointing out that the estimator for CF requires on the order of $N_{b}^{2} N^{2}$ operations where $N_{b}$ is the number of $\ell$-bands. One can get rid of the $N_{b}^{2}$ factor by rewriting it as an estimator for $C(\theta)$ in fine bins of $\theta$ and then Legendre-transforming the result, as was done in [7]. Further computational accelerations are possible by use of KD-tree search techniques which use coarsegraining at large distances [21,22]. In addition, fast spherical harmonic transforms lead to great time-savings in harmonic methods.

However, the simplicity of these other choices for $W$ does have drawbacks. Specifically, high-noise areas and low-noise areas make equal contribution to the estimator. To date, the success of these methods has only been demonstrated on simulations with homogeneous white noise. The first obvious improvement to $\mathrm{CF}$ is to replace $W_{i j}=\delta_{i j}$ with $W_{i j}=1 / \sigma_{i}^{2} \delta_{i j}$ (in pixel space) as suggested in [7].

What is less obvious is how to weight pixel pairs in the presence of correlated noise. This is where further development of the CF approach is most needed. One possible route to pursue is band-diagonal choices of $W_{i j}$ which capture the spatially-local noise correlations. Computation with band-diagonal $W$ 's can still be quite fast; they are still order of $N^{2}$ as long as the bandwidth is less than $\sqrt{N}$. Perhaps longer-range correlations could be included in some hybrid scheme of HD and CF. Here CF (with band-diagonal $W_{i j}$ ) would be used on the primary map and then HD would be used to calculate lower- $\ell$ values which may have been affected by long-range noise correlations. This hybrid scheme also has the advantage of complementing HD where its spectral resolution is lowest 23].

Although the calculation of $C_{l}$ is fast with simple choices for $W$, the calculation of the error covariance matrix (Eq. 21) is slow; i.e. the number of operations scales with $N^{3}$ because of the matrix multiplications. One option is to estimate the errors by Monte-Carlo methods [7]. Another is to combine the $\mathrm{CF}$ and $\mathrm{HD}$ approaches in yet another way: use CF as a means to produce an input power spectrum sufficiently close to the optimal one that only a single iteration of HD is required.

\section{CONCLUSIONS}

We have concentrated on developing a fast and reliable method for calculating power spectra and their uncertainties from maps with $N=10^{4}$ to $10^{5}$ pixels. Methods that work in this regime are of immediate practical importance. Our tests show very good agreement with exact methods at the lower end of our $N$ range where the exact analysis is feasible on a supercomputer. The HD method is the only existing method for calculating a power spectrum and its uncertainties from general, inhomogeneous correlated noise patterns with maps of this size in reasonable amounts of time 24].

We have not tested our method on maps with correlated noise. But since noise-correlations are taken into account exactly within each submap, we expect our method to handle correlated noise effectively, unlike the other fast methods mentioned above. These expectations will be put to the test soon as HD is applied to existing datasets from LDB flights, such as Archeops TopHat s. $^{2}$.

The local nature of the method has some advantages for controlling contamination of the final power spectrum result. In the extreme, one can simply cull submaps with the largest foreground contamination. Less drastically, one could down-weight the power spectrum determinations from submaps according to the suspected level of contamination.

To summarize, we have developed and investigated an HD method of power-spectrum estimation. We have demonstrated that for LDB-size maps HD is sufficiently fast and insignificantly sub-optimal. Its main advantages over other fast methods are its generality (including its ability to handle correlated noise) and the fact that the power spectrum uncertainties are calculated directly. Application to larger maps will rely on further approximations which we expect to work well but require further investigation. The main disadvantage to $\mathrm{HD}$ is the degraded spectral resolution at the smallest angular scales. The impact of this degradation on parameterdetermination also warrants further investigation. The combination of HD with other methods may be fruitful.

Madcumba, a Fortran 90 implementation of the HD procedure, will be made available for public use. Comments and questions should be directed to O. Doré at dore@iap.fr.

\section{ACKNOWLEDGMENTS}

O.D. is grateful to the UC Davis Cosmology group for a warm hospitality. LK is grateful to IAP for the same. We

\footnotetext{
${ }^{\ddagger}$ http://www-crtbt.polycnrs-gre.fr/archeops/

${ }^{\S}$ http://topweb.gsfc.nasa.gov/
} 
benefited from conversations with J. R. Bond, J. Borrill, F. Bouchet, A. Jaffe, R. Stompor, P. Koev, D. Vibert and R. Teyssier and the computer resources of S. Colombi and NERSC.

[1] E.g., A. Jaffe et al., Phys. Rev. Lett. 86, 3475-3479 (2000).

[2] L. Knox, Phys. Rev. D48, 3502 (1995); G. Jungman, M. Kamionkowski, A. Kosowsky, and D. Spergel 1996, Phys. Rev. D D54, 1332 (1996); J. R. Bond, G. Efstathiou, and M. Tegmark Mon. Not. Roy. Astron. Soc., 291, L33 (1997); D. Eisenstein, W. Hu and M. Tegmark, Astrophys. J. 504, 57L (1998).

[3] J. R. Bond, R. Crittenden, A. H. Jaffe and L. Knox, Computing in Science and Engineering, vol. 1, no. 2, 21 (1999).

[4] J. Borrill, Phys. Rev. D 59, 027302 (1999).

[5] O. Doré, R. Teyssier, F.R. Bouchet, D. Vibert, astro$\mathrm{ph} / 0101112$, see

also http://ulysse.iap.fr/download/mapcumba

[6] B.D. Wandelt, E. Hivon \& K. Górski, astro-ph/0008111; astro-ph/9808292

[7] I. Szapudi, S. Prunet, D. Pogosyan, A. Szalay and J.R. Bond, astro-ph/0010256

[8] S.P. Oh, D.N. Spergel and G. Hinshaw, Astrophys. J. 510, 551 (1999).

[9] B. Wandelt 2001, Proceedings of MPA/MPE/ESO Conference "Mining the Sky", July 31 - August 4, 2000, Garching, Germany, astro-ph/0012333, astro$\mathrm{ph} / 0012416$

[10] J.R. Bond, A.H. Jaffe \& L. Knox, Phys. Rev. D 57, 2117 (1998).

[11] These equations were independently derived as the optimal, unbiased quadratic estimator in M. Tegmark, Phys. Rev. D 55, 5895 (1997).

[12] This can also be understood as the usual problem of localizing simultaneously in position and momentum: M. Tegmark, Mon.Not.Roy.Astron.Soc. 280, 299-308 (1996).

[13] J. Borrill and P. Koev 2001, in preparation.

[14] Górski E.K., Hivon E., Wandelt B.D. in proceedings of the MPA/ESO Garching Conference 1998, eds Banday A.J., Sheth K. and L. Da Costa and http://www.eso.org/ kgorski/healpix/

[15] A. Jaffe, private communication.

[16] J.R. Bond, A.H. Jaffe \& L. Knox, Astrophys. J. 533, 19 (2000).

[17] U. Seljak \& M. Zaldarriaga, Astrophys. J. , 469, 437, 1996

[18] We tested the scaling with $n_{\text {proc }}$ by running Madcumba using up to 77 processors.

[19] We remind the reader that application of $\mathrm{HD}$ to megapixel and larger maps requires some way to calculate $N$ for the sub-maps from the time-ordered data. This could be accomplished by the method we briefly described in subsection IID, which will be described in more detail in 13.

[20] That the correlation-function approach can be regarded as a quadratic estimator with sub-optimal weighting was emphasized in 阿.

[21] S. Colombi et al. 2001, in preparation.

[22] A. Moore et al. 2001, Fast Algorithms and Efficient Statistics: N-point Correlation Functions, Proceedings of MPA/MPE/ESO Conference "Mining the Sky", July 31

- August 4, 2000, Garching, Germany, astro-ph/0012333

[23] The speed-up with KD-tree search techniques will also lead to some spectral resolution degradation.

[24] A possible exception is a monte-Carlo pseudo- $C_{l}$ method to be described in E. Hivon et al. 2001, in preparation. 